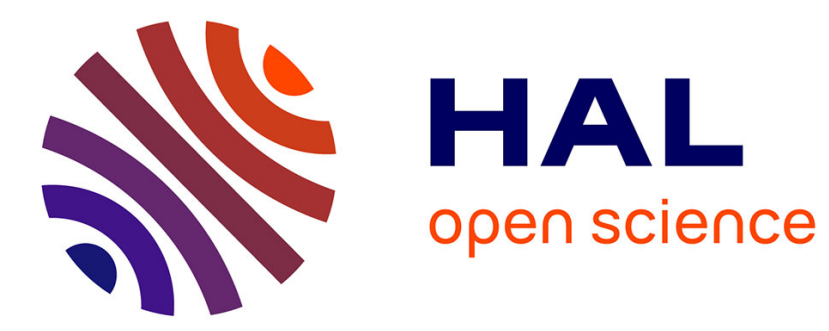

\title{
3D coronary structure tracking algorithm with regularization and multiple hypotheses in MRI
}

Jérôme Velut, Christine Toumoulin, Jean-Louis Coatrieux

\section{To cite this version:}

Jérôme Velut, Christine Toumoulin, Jean-Louis Coatrieux. 3D coronary structure tracking algorithm with regularization and multiple hypotheses in MRI. IEEE International Symposium on Biomedical Imaging: From Nano to Macro (ISBI 2010), Apr 2010, Rotterdam, Netherlands. pp.37-40, 10.1109/ISBI.2010.5490421 . inserm-00526973

\section{HAL Id: inserm-00526973 https://www.hal.inserm.fr/inserm-00526973}

Submitted on 19 Oct 2010

HAL is a multi-disciplinary open access archive for the deposit and dissemination of scientific research documents, whether they are published or not. The documents may come from teaching and research institutions in France or abroad, or from public or private research centers.
L'archive ouverte pluridisciplinaire HAL, est destinée au dépôt et à la diffusion de documents scientifiques de niveau recherche, publiés ou non, émanant des établissements d'enseignement et de recherche français ou étrangers, des laboratoires publics ou privés. 


\title{
3D CORONARY STRUCTURE TRACKING ALGORITHM WITH REGULARIZATION AND MULTIPLE HYPOTHESES IN MRI
}

\author{
Jérôme Velut, Christine Toumoulin and Jean-Louis Coatrieux \\ LTSI, Université de Rennes 1, Campus de Beaulieu, Bât 22, Rennes, F-35042 Cedex \\ INSERM, U642, Rennes, F-35000, France
}

\begin{abstract}
This paper presents an improved version of a tracking algorithm for the extraction of the 3D central axis of tubular-like object in a low-contrast and multi-object environment. This improvement concerns two aspects: (1) an efficient Infinite Impulse Response (IIR) filtering of the successive tracking directions is used to introduce regularization and (2) a multiple hypotheses testing procedure allows an almost exhaustive selection of the best centreline location by building different paths according to the applied parameters. A score is then computed for each hypothesis that helps in the selection of the best path along the tree. The improved tracking algorithm is tested on Magnetic Resonance Angiography (MRA) datasets to extract the coronary artery centrelines.
\end{abstract}

Index Terms - Spatial tracking, Multiple hypotheses, IIR, Regularization, Coronary MRA.

\section{INTRODUCTION}

Tracking of vascular features in images has been of interest for the past two decades in the field of medical imaging. It enables the extraction of 3D structures from volumes [1, 2, 3] enriching thus diagnosis and surgery plan with anatomical and functional quantitative data. Although these segmentation methods show promising results, they have been mainly tested on X-Ray angiography or multidetector computed tomography (MDCT) modalities [4]. Less attention has been paid to the extraction of coronary artery from magnetic resonance imaging (MRI). The appealing and fast evolving capabilities of MRI motivate the present work devoted to vessel extraction when no contrast agent is used.

This paper describes a spatial tracking algorithm that aims at extracting the central axis of tubular structures. It outlines as follows: its basic principle is given section 2. Improvements are then respectively described in sections 3 (regularization of the direction of the tracking process) and 4 (Application of a multiple hypothesis testing procedure in the vessel central axis research).The algorithm was applied to MRI coronary angiography (MRA) datasets for the extraction of coronary artery central axis. Section 5 reports on the results of the evaluation.

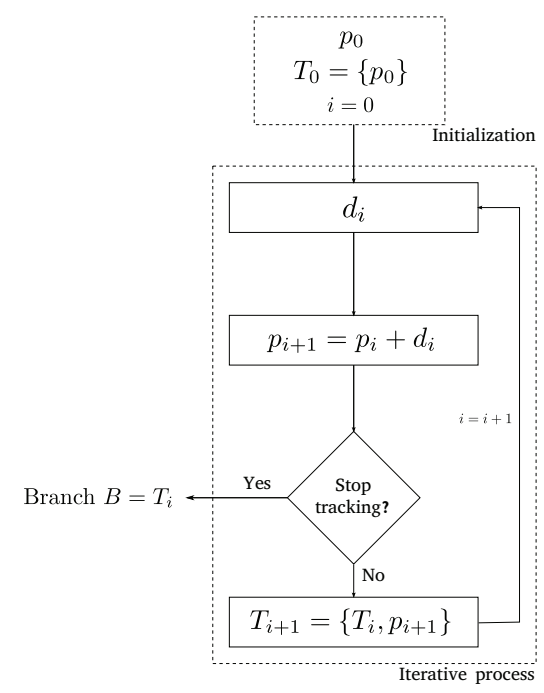

Fig. 1. Flow-chart of a generic spatial tracking algorithm. $p_{i}$ is the current point, $d_{i}$ is the estimated direction

\section{A DEFINITION OF SPATIAL TRACKING}

A spatial tracking algorithm aims at tracking a feature of interest in an image (2D or 3D) by assuming that some of its properties (based on edge, region or even shape similarities) smoothly evolve. Figure 1 shows a general iterative algorithm of spatial tracking that outputs a curve built from successive positions susceptible to correspond to the object feature under consideration. In this framework, the position $p$ at iteration $i+1$ is the translation of $p_{i}$ along the estimated direction $d_{i}$. $p_{i+1}$ will be appended to the current path (or track $T$ ). The output is a curve $B$ with simple topology, having no bifurcation (i.e. each point $p_{i}$ has two neighbours $p_{i-1}$ and $p_{i+1}$, except at extremities). The core equation is then:

$$
p_{i+1}=p_{i}+d_{i}
$$

where $p_{0}$ is a seed point, either user-defined or automatically extracted. Step length is included in $d_{i}$ through its norm. 


\section{REGULARIZATION OF ORIENTATIONS}

Regularization is often used in image analysis when noise falsefully influence the processing $[5,6]$. in [6] the regularization is conducted through a smoothing B-Spline filter. This filter has a symmetric fourth order IIR low-pass transfer function, which construction gives rise to a cascaded causal/anticausal implementation of a second order IIR filter called $S_{\lambda}^{+}$:

$$
S_{\lambda}^{+}(z)=\frac{b_{0}}{1+a_{1} \cdot z^{-1}+a_{2} \cdot z^{-2}}
$$

where $b_{0}, a_{1}$ and $a_{2}$ are the coefficients of the filter and $\lambda$ the regularization parameter controlling the degree of smoothing of the spline (refer to [6] for an analytic formulation). We define by $\hat{D}$ an $S_{\lambda}^{+}$-filtered signal in the z-space:

$$
\hat{D}(z)=S_{\lambda}^{+}(z) \cdot D(z)
$$

where $D(z)$ is the input signal. A recursive equation is derived from equations (2) and (3):

$$
\hat{d}_{i}=b_{0} \cdot d_{i}-a_{1} \cdot \hat{d}_{i-1}-a_{2} \cdot \hat{d}_{i-2}
$$

In the present work, we applied the filter to smooth the successive directions of the tracking process. Nevertheless, the temporal nature of signal required to only use the causal transfer function (2). Each component $\left(x_{i}, y_{i}, z_{i}\right)$ of the direction $d_{i}$ were considered as separate 1D signals and filtered through equation (4). Thus, equation (1) became:

$$
p_{i+1}=p_{i}+\hat{d}_{i}
$$

The recursive implementation offers a comprehensive range of regularization (from no regularization, $\lambda=0$ to high regularization $\lambda \rightarrow \infty$ ) with only three multiplications and three additions, thus preserving the fast nature of the iterative curve extraction algorithm (equation (5)).

\section{MULTIPLE HYPOTHESES FRAMEWORK}

According to [7], "Multiple hypothesis testing involves testing multiple hypotheses simultaneously; each hypothesis is associated with a test statistic". In [1], a template-based feature tracking algorithm builds a tree of possible paths at each iteration - the hypotheses. However, this implementation adds a search tree depth parameter and a predefined threshold for the best hypothesis selection. Here, we propose to reduce the tedious tuning of parameters by embedding the core curve extraction in a multiple hypothesis testing procedure.

\subsection{Hypothesis definition}

An hypothesis is defined as a vector of parameters $\rho=$ $\left(\rho_{0}, \ldots, \rho_{k}, \ldots\right)$ involved in the extraction method. The maximum number of tested hypotheses is:

$$
\operatorname{card}(\Omega)=\prod_{k=0}^{K-1} n_{k}
$$

\begin{tabular}{lcccc}
\hline Curve & $L(\lambda)$ & $L_{0}$ & $\frac{L_{0}}{L}$ & Seed location \\
\hline B1 & $65.59(0.9)$ & 28.15 & 0.43 & Medial RCA \\
B2 & $130.95(0.9)$ & 78.71 & 0.60 & Proximal LAD \\
B3 & $71.64(1.3)$ & 21.67 & 0.30 & Distal LAD \\
B4 & $355.86(0.9)$ & 86.18 & 0.24 & Proximal RCA \\
\hline
\end{tabular}

Table 1. Ratio between the non-regularized curve length $L_{0}(\lambda=0)$ and the longest extracted curve $L(\lambda), \lambda$ being the regularization rate associated with the latter.

where $K$ is the number of parameters (i.e. $\rho$ dimension), $n_{k}$ is the number of possible values that $\rho_{k}$ can take and $\Omega$ is the set of tested hypotheses, factually represented by a set of extracted curves.

When $\rho_{k}$ is defined on $\mathbb{R}$, its values are taken in a finite subset included in $\mathbb{R}$ with sampling step $\omega=\frac{\max \left(\rho_{k}\right)-\min \left(\rho_{k}\right)}{n_{k}}$

\subsection{Decision processes}

The number of extracted curves can be very high as it depends on the dimension of the vecteur $\rho$ (equation (6)). At the final stage, the problem comes to select the curves that represent at best the vascular structure. A score may be computed for each hypothesis that can be used in either an interactive or automatic selection of the best solutions. Integration variables are for instance the sampling step for length computation, image intensity in order to compute the brightness of a curve, or an eccentricity measure (such as the vesselness of Frangi et al. [8]).

\section{RESULTS}

We processed 4 datasets acquired on a 1.5 T MRA Philips system using a non-contrast breath-hold 3D steady-state free precession (SSFP) protocol [9]. Imaging was performed during mid-diastole, which is quiescent period in cardiac cycle. Spatial resolution was quite identical for all four sequences (in-plane resolution : $0.55 \times 0.55 \mathrm{~mm}$, slice distance : between 0.7 and $0.9 \mathrm{~mm}$, slice size : $512 \times 512$, number of slices: between 110 to 140 ).

We applied the geometrical moment-based tracking (GMT) algorithm, proposed in [2] and brought improvements to deal with MRA datasets (less contrasted than MSCT datasets): we introduced a regularization on the computation of the tracking direction to make it more robust against the noise and applied a multiple hypothesis procedure to build a set of possible paths (according to the applied parameters) along a vascular branch.

\subsection{Benefit of regularization}

We studied the impact of $\lambda$ on the result of centerline extraction for several coronary artery branches (Right Coronary Artery (RCA), IntraVentricular Artery (IVA), Circonflex Artery $(\mathrm{Cx}))$. The variation of $\lambda$ ranged between 0 and 1.6 


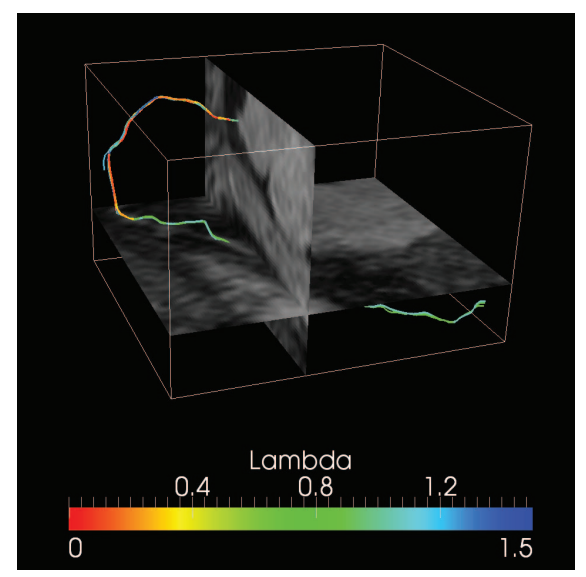

Fig. 2. 16 RCA curves were obtained from the same seed point (one curve per value of $\lambda$ ). These curves appears in colour (a colour is associated with a value of $\lambda$ ) and superposed in the image volume.

\begin{tabular}{cccccccc}
\hline & $\sigma$ & $\lambda$ & $r_{\min }$ & $r_{\max }$ & $L$ & $\bar{\nu}$ & $\frac{\bar{\nu}}{L}$ \\
\hline $\mathcal{C}_{0}$ & -1 & 0.4 & 0.85 & 0.90 & 145.3 & 41.76 & $\mathbf{0 . 2 9}$ \\
$\mathcal{C}_{1}$ & 1 & 0.6 & 0.75 & 0.95 & $\mathbf{4 0 0 . 6}$ & $\mathbf{8 3 . 3}$ & 0.20 \\
$\mathcal{C}_{2}$ & 1 & 0.8 & 0.75 & 0.95 & 320.8 & 63.5 & 0.19 \\
\hline
\end{tabular}

Table 2. Values of parameters for the selected curves. Selected curves are associated with the highest length normalized vesselness

and the incremental step was 0.1 ( $\lambda=0$ corresponds to no regularization). For a given branch, the tracking algorithm was initiated from an used defined seed point. A tracking process was then performed for each value of $\lambda$. In total, 16 curves were obtained per branch. Table 1 provides for each considered branch (RCA and IVA, for 2 datasets), the value of $\lambda$ which gives rise to the longest extracted curve $L(\lambda)$, this length when $\lambda=0\left(L_{0}\right)$ and the ratio $\left(\frac{L_{0}}{L}\right)$. Figure 2 shows the 16 centerlines extracted on one RCA branch, from the same seed point. For $\lambda=0$, the method acts like the original GMT algorithm and allows extracting the proximal and medial RCA segments. The regularization allows to reach the distal part of the RCA.

\subsection{Benefit of Multiple Hypothesis}

The original tracking algorithm considers 4 parameters :

- the seed points $p_{0}$

- the initial tracking direction $\sigma$

- the stopping criterion based on second order moment ratios $r_{\min }$ and $r_{\max }$

The stopping criterion parameter which is set empirically in the original algorithm, is here included in the vector of parameters $\rho$ to make $r_{\min }$ and $r_{\max }$ varying inside the multiple hypothesis framework. This vector of parameters $\rho$ is

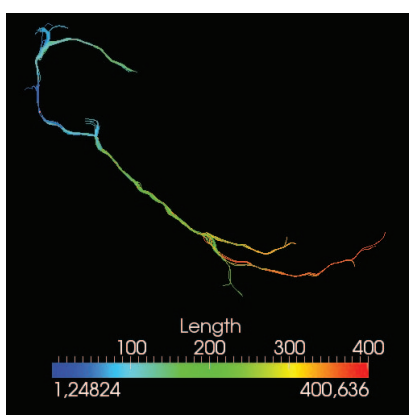

(a) Branch lengths $L$.

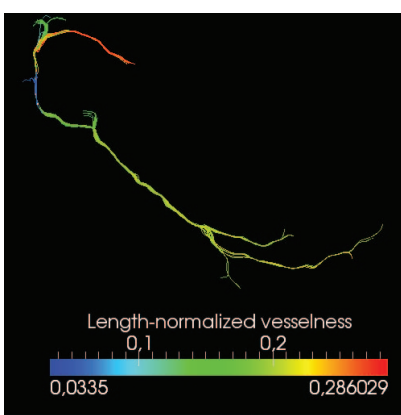

(c) Length-normalized vesselness $\frac{\bar{\nu}}{L}$.

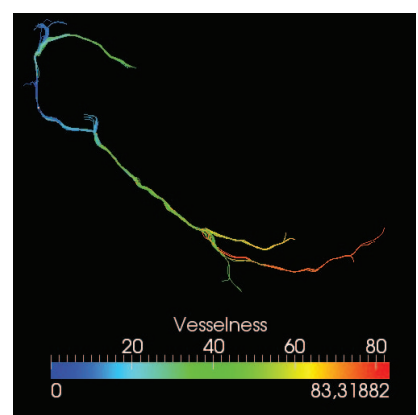

(b) Frangi's vesselness integrated over each curve $\bar{\nu}$.

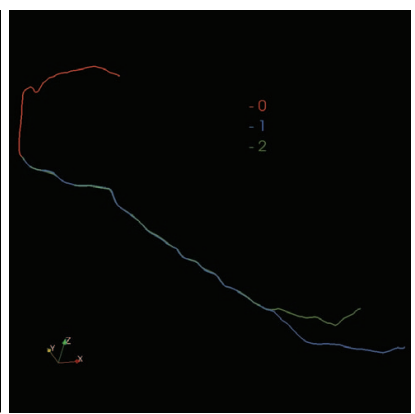

(d) Final RCA made from 3 curves $\mathcal{C}_{0}, \mathcal{C}_{1}$ and $\mathcal{C}_{2}$.
Fig. 3. 270 curves were extracted on the RCA branch. Each curve is coloured according to its length (a), vesselness measure (b) and lenght-normalized vesselness measure $(c)$. the final curve $(d)$ is the longest one associated with the highest measure of cylindricity.

completed with the regularization parameter $\lambda$, bringing thus this vector to 5 components:

$$
\rho=\left(p_{0}, \sigma, \lambda, r_{\min }, r_{\max }\right)
$$

with:

$$
\begin{aligned}
p_{0} & \\
\lambda \in[0,1.6], & \omega=0.4 \\
r_{\min } \in[0.75,1], & \omega=0.05 \\
r_{\max } \in\left[r_{\min }, 1\right], & \omega=0.05 \\
\sigma=\{-1,1\} &
\end{aligned}
$$

The multiple hypothesis strategy was applied to each branch (RCA, IVA and Cx). For one given branch, the algorithm was run for each different setting of the parameters (excepted the seed point $p_{0}$ which remained the same). Figure 3 depicts the result of this extraction on the RCA branch. In total, 270 curves were extracted. We designed then 3 scores to help in the best hypothesis selection: the curve length $L$ (figure 3(a)), the cumulated Frangi's vesselness $\bar{\nu}$ (figure 3(b)), the length-normalized vesselness that represents a combination of the two former measures (figure 3(c)). These scores were respectively exploited to select the longest curve associating the highest vesselness measure. Result is displayed in figure 3(d). The corresponding parameters and 


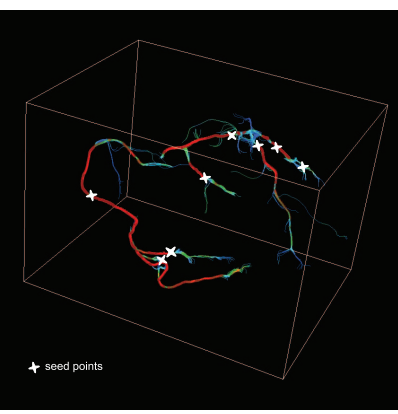

(a) Multiple hypothesis outputs. White crosses depict seed point locations.

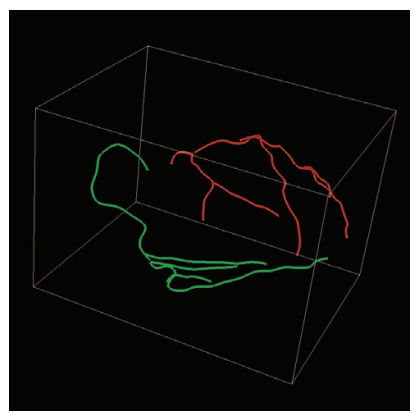

(b) Manual extraction. LCA (red) and RCA (green)

Fig. 4. Extraction of the coronary artery tree: (a) multiple hypothesis GMT algorithm, (b) manual extraction.

scores are given in table 2.

Curve $\mathcal{C}_{0}$ includes the proximal and medial RCA segments and was selected according to its length-normalized vesselness score. Curve $\mathcal{C}_{1}$ departs from the medial RCA segment, goes through the distal RCA and part of the right posterior atrioventricular segments. this curve represents the longest extracted one and is associated with the highest cumulated vesselness score. Curve $\mathcal{C}_{2}$ includes the distal RCA segment and continues in the right posterior descending artery segment. It has been selected manually based on anatomical knowledge. The computation time amounted to 34 seconds, on a single core processor, for processing one branch.

Figure 4 displays the coronary artery tree extracted from the multiple hypothesis-based tracking algorithm. Parameter vector $\rho$ was defined as in (7). 9 seed points were interactively distributed over the vascular structure. 7167 curves were extracted. The computation time amounted to $11 \mathrm{~m} 10 \mathrm{~s}$. The extracted vascular tree can be compared with the manual extraction (figure 4(b)).

\section{CONCLUSION}

we have proposed a regularization process for the vessel orientation computation and a multiple hypothesis testing procedure that significantly improves the results of the artery centreline extraction.

Although the selection of the best hypothesis was done manually, it relied on different scores that can be exploited in an automatic way. Further improvements will be made in that direction.

A consequence of multiple hypothesis testing procedure is its high computational cost. However, this is moderating thanks to existing dependences between parameters and prior knowledge. Furthermore, each centreline computation being completely independent, a parallel implementation is possible that could significantly decreases the computation time.

Results on coronary MRA data are promising enough to

justify further quantitative investigations and comparisons to other methods.

\section{ACKNOWLEDGEMENTS}

The research leading to these results has received funding from the European Community's Seventh Framework Programme (FP7/2007-2013) under grant agreement $\mathrm{n}^{\circ} 224495$ (euHeart project). We are also grateful toward Olivier Ecabert and Juergen Weese from Philips Research Aachen for providing the MRA data sets.

\section{REFERENCES}

[1] O. Friman, M. Hindennach, and H.-O. Peitgen, "Template-based multiple hypotheses tracking of small vessels," in IEEE ISBI, 2008, pp. 1047-1050.

[2] A. Larralde, C. Boldak, M. Garreau, C. Toumoulin, D. Boulmier, and Y. Rolland, "Evaluation of a 3D segmentation software for the coronary characterization in multi-slice computed tomography," in FIMH, 2003, pp. $39-51$.

[3] C. Toumoulin, C. Boldak, J. L. Dillenseger, J. L. Coatrieux, and Y. Rolland, "Fast detection and characterization of vessels in very large 3-D data sets using geometrical moments," IEEE Transactions on Biomedical Engineering, vol. 48, no. 5, pp. 604-606, 2001.

[4] M. Schaap et al., "Standardized evaluation methodology and reference database for evaluating coronary artery centerline extraction algorithms.," Med Image Anal, vol. 13, no. 5, pp. 701-714, Oct 2009.

[5] J. Velut, H. Benoit-Cattin, and C. Odet, "Locally regularized smoothing B-snake," EURASIP Journal on Advances in Signal Processing, vol. 2007, pp. Article ID 76241, 12 pages, 2007, doi:10.1155/2007/76241.

[6] M. Unser, A. Aldroubi, and M. Eden, "B-spline signal processing. Part I. Theory," IEEE Transactions on Signal Processing, vol. 41, no. 2, pp. 821-833, 1993.

[7] G. Kang, K. Ye, N. Liu, D. B. Allison, and G. Gao, "Weighted multiple hypothesis testing procedures," Stat Appl Genet Mol Biol, vol. 8, no. 1, pp. Article23, 2009.

[8] A. F. Frangi, W. J. Niessen, K. L. Vincken, and M. A. Viergever, "Multiscale vessel enhancement filtering," in MICCAI, 1998, pp. 131-137.

[9] J. Peters, O. Ecabert, C. Meyer, H. Schramm, R. Kneser, A. Groth, and J. Weese, "Automatic whole heart segmentation in static magnetic resonance image volumes," in MICCAI, 2007, pp. 402-410. 